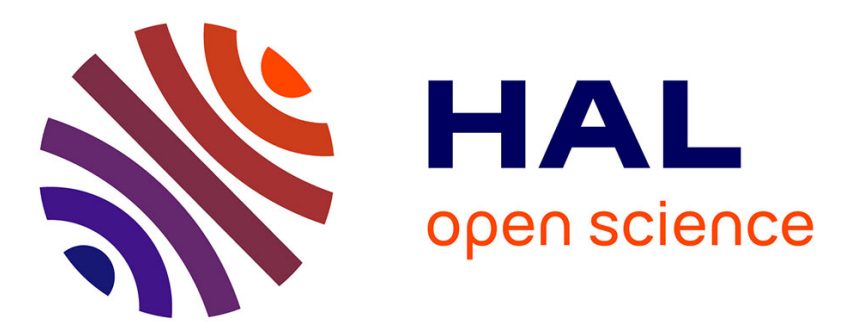

\title{
An allosteric transition trapped in an intermediate state of a new kinesin-inhibitor complex
}

Hung Yi Kristal Kaan, Venkatasubramanian Ulaganathan, David D. Hackney, Frank Kozielski

\section{- To cite this version:}

Hung Yi Kristal Kaan, Venkatasubramanian Ulaganathan, David D. Hackney, Frank Kozielski. An allosteric transition trapped in an intermediate state of a new kinesin-inhibitor complex. Biochemical Journal, 2009, 425 (1), pp.55-60. 10.1042/BJ20091207 . hal-00479242

\section{HAL Id: hal-00479242 \\ https://hal.science/hal-00479242}

Submitted on 30 Apr 2010

HAL is a multi-disciplinary open access archive for the deposit and dissemination of scientific research documents, whether they are published or not. The documents may come from teaching and research institutions in France or abroad, or from public or private research centers.
L'archive ouverte pluridisciplinaire HAL, est destinée au dépôt et à la diffusion de documents scientifiques de niveau recherche, publiés ou non, émanant des établissements d'enseignement et de recherche français ou étrangers, des laboratoires publics ou privés. 


\title{
An allosteric transition trapped in an intermediate state of a new kinesin- inhibitor complex
}

\author{
Hung Yi Kristal Kaan*, Venkatasubramanian Ulaganathan*, David D. Hackney ${ }^{\dagger}$ \& \\ Frank Kozielski ${ }^{*}$ \\ "The Beatson Institute for Cancer Research, Switchback Road, Bearsden, Glasgow \\ G61 1BD, Scotland, UK \\ tDepartment of Biological Sciences, Carnegie Mellon University, 4400 Fifth Avenue, \\ Pittsburgh, PA 15213, USA \\ ‡Correspondence: f.kozielski@beatson.gla.ac.uk
}

\begin{abstract}
Abbreviations used: STLC, S-trityl-L-cysteine; ATP, Adenosine triphosphate; ADP, Adenosine diphosphate; AMPPNP, 5'-adenylyl- $\beta, \gamma$-imidodiphosphate; FRET, Fluorescence resonance energy transfer; RNAi, RNA inteiference; $\mathrm{Mg}^{2+}$, Magnesium ion; PIPES, Piperazine-1,4-bis(2-ethanesulfonic acid); PDB, Protein Data Bank; DTT, Dithiothreitol; EGTA; Ethylene glycol tetraacetic acid; $A$, angstrom; cryoEM, cryoelectron microscopy.
\end{abstract}

\begin{abstract}
Human kinesin Eg5 plays an essential role in mitosis by separating duplicated centrosomes and establishing the bipolar spindle. Eg5 is an interesting drug target for the development of cancer chemotherapy, with 7 inhibitors already in clinical trials. Here, we report the crystal structure of the Eg5 motor domain complexed with a potent antimitotic inhibitor S-trityl-L-cysteine to $2.0 \AA$ resolution. The Eg5-STLC complex crystallises in space group $P 3_{2}$ with 3 molecules per asymmetric unit. Two of the molecules reveal the final inhibitor-bound state of Eg5, whereby Loop L5 has swung downwards to close the inhibitor-binding pocket, helix $\alpha 4$ has rotated by about $15^{\circ}$ and the neck-linker has adopted a docked conformation. The third molecule, however, revealed an unprecedented intermediate state, whereby local changes at the inhibitor-binding pocket have not propagated to structural changes at the switch II cluster and neck-linker. This provides structural evidence for the sequence of druginduced conformational changes.
\end{abstract}

\section{INTRODUCTION}

Human Eg5 is a member of the Kinesin-5 subfamily and is responsible for the formation of the bipolar mitotic spindle [1]. Current models suggest that the Eg5 homotetramer separates the duplicated centrosomes by pushing antiparallel microtubules apart [2,3]. The inhibition of Eg5 by RNAi, specific inhibitors, or immunodepletion causes cells to arrest in mitosis with monoastral spindles. $[1,4,5]$. Prolonged mitotic arrest then leads to the activation of the mitotic checkpoint and subsequent apoptotic cell death in certain cancer cell lines. Thus, Eg5 is an interesting candidate for drug development in cancer chemotherapy.

Cell-based and in vitro assays have led to the discovery of a series of inhibitors specific to Eg5. S-trityl-L-cysteine (STLC) was previously identified as a new, potent Eg5 inhibitor [6] and subsequent work revealed that STLC is a reversible, tightbinding inhibitor that does not compete with either ATP or microtubules [7], but slows down ADP release. In recent years, several crystal structures of Eg5 in complex with 
inhibitors have surfaced. When compared with the native structure of Eg5 without inhibitor [8], these crystal structures reveal several important conformational changes at key structural elements upon inhibitor binding. These conformational changes, which are observed in virtually all the inhibitor-bound Eg5 motor domain crystal structures, include the downward swing of the Loop L5 to close the inhibitor-binding pocket and also a shift in switch I [9]. In addition, helix a4 of the switch II cluster rotates by about $20^{\circ}$, on the plane of the Eg5 motor-microtubule interface, as shown by cryoEM reconstruction of the motor-microtubule complex [10]. This new position of the switch II cluster is also described as the "permissive" conformation, as this rearrangement opens up space for the neck-linker to dock onto the motor domain [11].

A conformational change in the neck-linker, which links the motor domain to the stalk domain, is believed to be pivotal in producing movement for the molecular motor. This highly conserved neck-linker is inherently flexible and mobile. In the ADP-bound conventional kinesin structure, the neck-linker is observed to be disordered [12] and undocked [13], but becomes ordered and docked in the microtubule-bound kinesinAMPPNP (slowly-hydrolyzable ATP analogue) structure [14]. Similarly, in the ADPbound Eg5 motor domain native structure, the neck-linker is ordered, undocked and perpendicular to the motor domain [8]. To date, all inhibitor-bound Eg5 motor domain structures reveal a docked neck-linker conformation, parallel to the motor domain.

From static images of the native structure and the final inhibitor-bound state, it was unclear, how these conformational changes were induced by inhibitor binding and what the sequence of these conformational changes was. Studies using FRET (fluorescence resonance energy transfer) have been carried out to gain insight into the intermediate transition states. Kinetic studies by Maliga et al. propose a model in which, following Monastrol binding to Eg5 motor domain, the downward swing of Loop L5 occurs first, followed by conformational changes in the switch II cluster, which allows the neck-linker to dock [15].

In this study, we report the crystal structure of an unprecedented intermediate state of the Eg5-STLC complex, which provides structural evidence for the sequence of structural changes that occur upon inhibitor binding.

\section{RESULTS \& DISCUSSION}

\section{Overall structure}

The crystal structure of the Eg5-STLC complex was solved at a resolution of $2.0 \AA$. Data collection and refinement statistics are shown in Table 1. The Eg5-STLC complex crystallises with three molecules (subsequently named $A, B$ and $C$ ) in the asymmetric unit. The final model comprises of residues Asn18 to Asn271 and Asn287 to Glu364 for molecules A (Fig 1A) and B; and Lys17 to Asn271 and Asn289 to Pro363 for molecule C. Loop L11 is missing as in most other kinesin structures. All three molecules have one molecule of $\mathrm{Mg}^{2+} \mathrm{ADP}$ bound in the nucleotide-binding pocket with the magnesium coordinated octahedrically with the $\beta$-phosphate, the oxygen (OG1) of the hydroxyl side chain of Thr112 and four water molecules. The inhibitor-binding pocket, bordered by helix $\alpha 2$, Loop L5 and helix $\alpha 3$, is occupied by the inhibitor STLC, which has a very well-defined electron density (Fig 1B). 


\section{Interactions in the inhibitor-binding pocket}

The conformation of STLC and the interactions between STLC and Eg5 are virtually identical in all three molecules. The three phenyl rings in STLC, which represent the hydrophobic part of the inhibitor, are buried in the allosteric site and display several hydrophobic interactions with the alkyl side chains of Glu215, Glu116 and Arg119 (Fig 2). We also observed edge-to-face (T-shape) interaction between the first phenyl group of STLC and the phenyl ring of Trp127; C-H---m interaction between the first phenyl group of STLC and the pyrrolidine ring of Pro137; offset stacked $\pi-\pi$ interaction between the second phenyl group and Tyr211; and $\mathrm{C}-\mathrm{H}---\pi$ interaction between the third phenyl group and the side chain of Leu214 [16]. On the other hand, the hydrophilic part of the inhibitor, namely the cysteine moiety, is exposed to the solvent and we observed several hydrogen bond interactions: the amino group of the cysteine moiety with the main chain carbonyl oxygen of Gly117, the side chain oxygen (OE1) of Glu116, and a structural water molecule; an oxygen (OXT) of the carboxyl group of the cysteine moiety with one of the side chain amino groups $(\mathrm{NH} 1)$ of Arg221 and four structural water molecules; and the other oxygen of the carboxyl group with one structural water molecule. (All structural water molecules mentioned are conserved in all three molecules of the asymmetric unit) Thus, our crystal structure reveals several important interactions in the inhibitor-binding pocket and helps to corroborate the mutagenesis studies, conducted by Brier et al. [17], which identified the residues that are essential for the inhibition of Eg5 by STLC.

\section{Conformational changes at the inhibitor-binding pocket}

The three Eg5-STLC molecules in the asymmetric unit reveal a variety of structural differences throughout the molecule, when compared with the native structure of the Eg5 motor domain [8] in the absence of any bound inhibitor. The most obvious proximal changes occur in the inhibitor-binding pocket. In all three molecules, we observed that Arg119 and Tyr211 move outwards, while Trp127 of the Loop L5 region (Gly117 to Ala133) swings downwards, by about $7 \AA$, from a solvent-exposed region to form an aromatic edge-to-edge $\pi$-interaction with the phenol ring of Tyr211 (Fig 2, Movie 1). These conformational changes are consistent with those observed in the Eg5-Monastrol complex [9]. These conformational changes also support the induced-fit model of the binding of the inhibitor to the Eg5 motor domain: Arg119 and Tyr211 move outwards to accommodate the inhibitor, while Trp127 swings downwards to close the entrance of the induced-fit pocket and forms a snug inhibitorbinding pocket. We also observed that residues Pro121 to Glu124 of molecule A are displaced by a maximum of $2 \AA$ away from the core of the motor domain, as compared to that of molecules $\mathrm{B}$ and $\mathrm{C}$. Upon close examination, we saw that crystal contacts between these residues in molecule $A$ and a symmetry-related molecule could have caused this small displacement.

\section{P-loop, helix $\alpha 3$ and switch I}

Another important region in the Eg5 motor domain is the catalytic site or nucleotidebinding pocket. In the native and STLC-bound structures, $\mathrm{Mg}^{2+} \mathrm{ADP}$ occupies the active site through binding of the $\alpha$ - and $\beta$-phosphates to the P-loop (residues Gln106 to Gly110). The overlay of molecules A, B and C with the native structure showed no differences in conformation of the P-loop. We then investigated the helix $\alpha 3$. In all three molecules, the apex of the helix $\alpha 3$ is shifted towards the solvent by a maximum of about $2 \AA$ at Glu209, when compared to the native structure (Fig 3B, 
Movie 2). This is probably to accommodate the inhibitor, since several residues of the helix $\alpha 3$ participate in the formation of the inhibitor-binding pocket. Following helix $\alpha 3$ is switch I (residues Asn229 to His236), which is thought to be a y-phosphate sensor. We observed that the conformation of all three molecules are identical, but they differ from that of the native structure in that the Loop L9 is shifted towards the solvent by about $6 \AA$ and displays an additional one-turn of a helix. The hydroxyl group of Tyr231, which is originally solvent-exposed in the native structure, moves by about $12 \AA$ into the hydrophobic environment of the protein.

\section{Intermediate state of switch II cluster and neck-linker}

Conformational changes at and around the inhibitor-binding pocket are thought to be translated to conformational changes at the switch II cluster (helix $a 4$, Loop L12, and helix a5) and the neck-linker region at the back of the Eg5 motor domain (Fig 3C, Movie 3). We overlaid molecules $A, B$ and $C$, using the main chain $C_{\alpha}$ atoms from residues 19 to 363 , and found that molecules $A$ and $B$ were almost identical with a root mean square deviation of $0.33 \AA$. However, we observed a larger mean deviation of $1.32 \AA$ when we overlaid molecules $A$ and $B$ with $C$. Upon closer examination of molecule $C$, we believe that we have trapped an intermediate state of inhibitor-bound Eg5, never seen before in any kinesin crystal structures. Henceforth, the structures of molecules $A$ and $B$, which have similar conformations, will be referred to as the final inhibitor-bound state, while the structure of molecule $C$ will be referred to as the intermediate state. The occurrence of this intermediate state, in particular the structural differences at the switch $\|$ cluster and the neck-linker, is not caused by crystal contacts with the symmetry-related molecule and is not a crystallographic artifact (Supplementary figure 1). The B-factors for the switch II cluster and neck-linker region, though higher than the average B-factor of the entire molecule, are consistent throughout molecules A, B and C. In fact, trapping of structural intermediates is possible in flexible regions of the kinesin motor in the absence of microtubules, because such structural changes would require low energy input [8]. We observed a clear progression of the switch II cluster as it rotates about $15^{\circ}$ from the native structure to the intermediate state and eventually to the final inhibitor-bound state. In fact, this progression can be seen as two distinct movements: first an anti-clockwise rotation of approximately $10^{\circ}$ about the origin around residue Leu293, then a similar rotation of approximately $5^{\circ}$ about an origin beyond the apex of helix $a 4$. The rearrangement of the switch II cluster opens up space for the neck-linker to adopt a docked position in the final inhibitor-bound state. In both the native and intermediate structures, the neck-linker, which connects the motor to the stalk domain of Eg5, is in an ordered, albeit undocked position, perpendicular to the helix a6. The neck-linker eventually moves by about $32 \AA$ to adopt a docked position, parallel to helix $\alpha 6$ in the final inhibitor-bound state. Thus, the structural intermediate found in molecule $\mathrm{C}$ reflects a dynamic switch II cluster and a flexible neck-linker region. Such flexibility in the neck-linker serves to emphasize its role in force generation and motility.

\section{Pathway of structural changes}

Although several crystal structures of Eg5 in complex with inhibitors have been deposited in the Protein Data Bank, all of them exhibit the same conformation and structural changes representative of the final state of the inhibitor-bound Eg5. As the crystal structures are static images, it remains unclear how these structural changes come about and the sequence of events leading to the final inhibitor-bound state. Studies using FRET have been carried out to gain insight into the intermediate 
transition states [15, 18]. By monitoring the quenching of Trp127 by Monastrol binding to Eg5 and using FRET, between mant-ADP at the active site and an Oregon green fluorophore attached to an introduced cysteine near the end of the neck-linker, Maliga et al. observed drug-induced biphasic conformational change of the Eg5 motor domain [15]. The first rapid phase involves conformational changes at Trp127 and other residues in the inhibitor-binding site. A much slower subsequent conformation change then results in the docking of the neck-linker onto the motor domain. The conformational changes observed in the intermediate state is consistent with that expected after initial inhibitor binding - siginificant local changes at the inhibitor-binding pocket - but before the docking of the neck-linker. Significantly, the changes in the intermediate state are moving in the direction of the final state. Complete conversion only occurs in the final inhibitor-bound state, where the necklinker becomes docked. Therefore, we propose that the binding of STLC to Eg5 causes drug-induced structural transition in 3 steps: first, Loop L5 swings downwards to close the inhibitor-binding pocket, which then translates to a rearrangement of the switch II cluster to adopt a "permissive" conformation, and finally the neck-linker docks onto the motor domain.

\section{MATERIAL AND METHODS}

\section{Protein expression and purification}

The motor domain of human Eg5 (residues 1-368) was initially cloned into the vector pET28a [19]. It was then cut with restriction enzymes Ncol and Xhol (NEB) and cloned into a modified pProExHT expression plasmid (Invitrogen), yielding a Precision Protease-cleavable N-terminal hexahistidine-tagged protein. The plasmid was transformed into E. coli BL21 (DE3) pLysS (Invitrogen). Three liters of bacterial culture were grown at $37^{\circ} \mathrm{C}$ in TB (Terrific Broth) supplemented with $100 \mathrm{mg} / \mathrm{l}$ Ampicillin to an $A_{600}$ of approximately 0.7 and induced overnight with $0.5 \mathrm{mM}$ isopropyl ß-D-thiogalactopyranoside (Melford). Harvested cells were resuspended in buffer A (50 mM PIPES pH 6.8, $2 \mathrm{mM} \mathrm{MgCl}_{2}, 250 \mathrm{mM} \mathrm{NaCl}, 20 \mathrm{mM}$ Imidazole, $1 \mathrm{mM}$ PMSF, $1 \mathrm{mM} \mathrm{MgATP}$, and $2.5 \mathrm{mg}$ lysozyme) and subjected to one cycle of freezethaw and sonication, before $1 \mathrm{mM}$ DNasel and $10 \mathrm{mM} \mathrm{MgCl}$ were added.

Clear lysate was loaded onto a HisTrapFF column (GE Healthcare) equilibrated in buffer $A$. The resin was washed with buffer A containing $50 \mathrm{mM}$ Imidazole and the proteins were eluted on a gradient of buffer A from $50 \mathrm{mM}$ to $500 \mathrm{mM}$ Imidazole. Fractions containing protein of interest were pooled and the buffer was exchanged for protease buffer ( $50 \mathrm{mM}$ PIPES pH 6.8 and $250 \mathrm{mM} \mathrm{NaCl}$ ). Precision Protease, 1 $\mathrm{mM} \mathrm{MgATP}$, and $2 \mathrm{mM} \mathrm{MgCl}_{2}$ were added and reactions were incubated overnight at $4^{\circ} \mathrm{C}$. Uncleaved proteins and protease were removed by NiNTA affinity chromatography. The cleaved protein was subjected to size exclusion chromatography on a Superdex 75 column (GE Healthcare) equilibrated in gel filtration buffer (50 mM PIPES pH 6.8, $50 \mathrm{mM} \mathrm{KCl,} 1 \mathrm{mM}$ DTT, $1 \mathrm{mM}$ EGTA, and 1 $\mathrm{mM} \mathrm{MgCl} 2$ ). Fractions containing protein of interest were pooled and concentrated on an Amicon Ultra filtration device (Millipore) to a final concentration of approximately $20 \mathrm{mg} / \mathrm{ml}$, frozen in liquid nitrogen, and stored at $-80^{\circ} \mathrm{C}$.

\section{Crystallisation of the Eg5-STLC complex}

Purified Eg5 was incubated with excess powdered STLC (Novabiochem) overnight at $4^{\circ} \mathrm{C}$. Before setting up of crystal trays, the sample was centrifuged at $13,000 \mathrm{rpm}$ for 
5 min at $4^{\circ} \mathrm{C}$ to pellet undissolved inhibitor. Crystals of Eg5 with STLC appeared after 1 month in hanging drops by mixing $2.5 \mu \mathrm{l}$ of protein-inhibitor complex $(10 \mathrm{mg} / \mathrm{ml})$ with $2.5 \mu \mathrm{l}$ of reservoir solution containing $22 \%$ polyethylene glycol-3350, $0.15 \mathrm{M}$ Sodium tartrate dibasic dihydrate, and 0.1 M MES pH 6.5 in VDX plates (Hampton Research) at $4^{\circ} \mathrm{C}$. A block-shaped crystal with dimensions of approximately $0.1 \times 0.1$ x $0.3 \mathrm{~mm}$ was immersed in cryoprotectant solution (24\% polyethylene glycol-3350, $0.18 \mathrm{M}$ Sodium tartrate dibasic dihydrate, $0.12 \mathrm{M} \mathrm{MES} \mathrm{pH} \mathrm{6.5,} 0.06 \mathrm{M} \mathrm{KCl}$ and $20 \%$ erythritol) and flash frozen in liquid nitrogen.

\section{Data collection and processing}

Diffraction data were recorded at the European Synchrotron Radiation Facility (ESRF) on station ID23-1. The crystals belonged to space group $P 3_{2}$. Data were processed using iMosflm [20] and SCALA from the CCP4 suite of programs [21] . The structure was solved by molecular replacement (MOLREP) using one molecule of Eg5 (PDB code 1X88) as a search model. Three subunits were positioned and refined with REFMAC5 [22]. The calculation of $R_{\text {free }}$ used $5 \%$ of data. Electrondensity and difference density maps all $\sigma_{A}$-weighted, were inspected and the model was improved using Coot [23]. The coordinates and the cif dictionary for the inhibitor molecule were calculated using Dundee PRODRG server [24]. Crystallographic statistics are given in Table 1 . Model geometry was analysed using PROCHECK [25]. $96.49 \%(880)$ of the residues are in the preferred regions, $2.30 \%(21)$ are in the allowed regions, and $1.21 \%$ (11) are outliers as shown by the Ramachandran Plot. Figures are prepared using PyMOL [26].

Since $3^{\text {rd }}$ generation radiation was used for data collection, the crystal suffered slight radiation damage. The refined difference fourier map showed some negative density around the sulphur atom of STLC in all three molecules and also around the sulphur atoms of other residues at the surface. As sulphur atoms absorb more photons, at wavelength of about $1 \AA$, compared to other heavy atoms $(C, O \& N)$ present in the protein, the radiation damage is greater compared to that of the other atoms. Moreover, sulphur atoms that are more exposed (present at the surface) or are in close proximity to solvent water molecules experience more profound effect due to secondary radiation damage from the water molecules. [27, 28]

As the space group $P 3_{2}$ is prone to crystal twinning, we carefully examined the cumulative distribution function in ctruncate and found no indication of twinning [29]. In addition, we submitted the data to the Merohedral Crystal Twinning Server [30], which also confirmed the absence of any significant twinning.

\section{COORDINATE FILES}

Coordinates and structure factors for the Eg5-STLC complex (PDB ID: 2wog) were deposited at the Protein Data Bank.

\section{ACKNOWLEDGEMENTS}

We thank David Flot for his support at the ESRF beamline ID23-1. Kristal Kaan holds a National Science Scholarship, financed by $A^{*}$ STAR (Singapore), and this publication contains part of her doctoral thesis. We thank CR-UK and the National Science Foundation (Grant 0615549) for financial support. 


\section{REFERENCES}

1 Blangy, A., Lane, H. A., d'Herin, P., Harper, M., Kress, M. and Nigg, E. A. (1995) Phosphorylation by p34cdc2 regulates spindle association of human Eg5, a kinesin-related motor essential for bipolar spindle formation in vivo. Cell. 83, 11591169

2 Sawin, K. E., LeGuellec, K., Philippe, M. and Mitchison, T. J. (1992) Mitotic spindle organization by a plus-end-directed microtubule motor. Nature. $359,540-543$

3 Cole, D. G., Saxton, W. M., Sheehan, K. B. and Scholey, J. M. (1994) A "slow" homotetrameric kinesin-related motor protein purified from Drosophila embryos. J Biol Chem. 269, 22913-22916

$4 \quad$ Weil, D., Garcon, L., Harper, M., Dumenil, D., Dautry, F. and Kress, M. (2002) Targeting the kinesin Eg5 to monitor siRNA transfection in mammalian cells. Biotechniques. 33, 1244-1248

5 Mayer, T. U., Kapoor, T. M., Haggarty, S. J., King, R. W., Schreiber, S. L. and Mitchison, T. J. (1999) Small molecule inhibitor of mitotic spindle bipolarity identified in a phenotype-based screen. Science. 286, 971-974

6 DeBonis, S., Skoufias, D. A., Lebeau, L., Lopez, R., Robin, G., Margolis, R. L., Wade, R. H. and Kozielski, F. (2004) In vitro screening for inhibitors of the human mitotic kinesin Eg5 with antimitotic and antitumor activities. Mol Cancer Ther. 3, 1079-1090

7 Skoufias, D. A., DeBonis, S., Saoudi, Y., Lebeau, L., Crevel, I., Cross, R., Wade, R. H., Hackney, D. and Kozielski, F. (2006) S-trityl-L-cysteine is a reversible, tight binding inhibitor of the human kinesin Eg5 that specifically blocks mitotic progression. J Biol Chem. 281, 17559-17569

8 Turner, J., Anderson, R., Guo, J., Beraud, C., Fletterick, R. and Sakowicz, R. (2001) Crystal structure of the mitotic spindle kinesin Eg5 reveals a novel conformation of the neck-linker. J Biol Chem. 276, 25496-25502

9 Yan, Y., Sardana, V., Xu, B., Homnick, C., Halczenko, W., Buser, C. A., Schaber, M., Hartman, G. D., Huber, H. E. and Kuo, L. C. (2004) Inhibition of a mitotic motor protein: where, how, and conformational consequences. J Mol Biol. $335,547-554$

10 Krzysiak, T. C., Wendt, T., Sproul, L. R., Tittmann, P., Gross, H., Gilbert, S. P. and Hoenger, A. (2006) A structural model for monastrol inhibition of dimeric kinesin Eg5. EMBO J. 25, 2263-2273

11 Marx, A., Muller, J. and Mandelkow, E. (2005) The structure of microtubule motor proteins. Adv Protein Chem. 71, 299-344

12 Kuil, F. J., Sablin, E. P., Lau, R., Fletterick, R. J. and Vale, R. D. (1996) Crystal structure of the kinesin motor domain reveals a structural similarity to myosin. Nature 380, 550-555

13 Sindelar, C. V., Budny, M. J., Rice, S., Naber, N., Fletterick, R. and Cooke, R. (2002) Two conformations in the human kinesin power stroke defined by X-ray crystallography and EPR spectroscopy. Nat Struct Biol. 9, 844-848 
14 Rice, S., Lin, A. W., Safer, D., Hart, C. L., Naber, N., Carragher, B. O., Cain, S. M., Pechatnikova, E., Wilson-Kubalek, E. M., Whittaker, M., Pate, E., Cooke, R., Taylor, E. W., Milligan, R. A. and Vale, R. D. (1999) A structural change in the kinesin motor protein that drives motility. Nature. 402, 778-784

15 Maliga, Z., Xing, J., Cheung, H., Juszczak, L. J., Friedman, J. M. and Rosenfeld, S. S. (2006) A pathway of structural changes produced by monastrol binding to Eg5. J Biol Chem. 281, 7977-7982

16 Chakrabarti, P. and Bhattacharyya, R. (2007) Geometry of nonbonded interactions involving planar groups in proteins. Prog Biophys Mol Biol. 95, 83-137

17 Brier, S., Lemaire, D., Debonis, S., Forest, E. and Kozielski, F. (2004) Identification of the protein binding region of S-trityl-L-cysteine, a new potent inhibitor of the mitotic kinesin Eg5. Biochemistry. 43, 13072-13082

18 DeBonis, S., Simorre, J. P., Crevel, I., Lebeau, L., Skoufias, D. A., Blangy, A., Ebel, C., Gans, P., Cross, R., Hackney, D. D., Wade, R. H. and Kozielski, F. (2003) Interaction of the mitotic inhibitor monastrol with human kinesin Eg5. Biochemistry. 42, 338-349

19 Garcia-Saez, I., DeBonis, S., Lopez, R., Trucco, F., Rousseau, B., Thuery, P. and Kozielski, F. (2007) Structure of human Eg5 in complex with a new monastrolbased inhibitor bound in the R configuration. J Biol Chem. 282, 9740-9747

20 Leslie, A. G. W. (1992) Joint CCP4 + ESF-EAMCB Newsletter on Protein Crystallography. No. 26

21 Collaborative Computational Project, N. (1994) The CCP4 Suite: Programs for Protein Crystallography. Acta Cryst, 760-763

22 Murshudov, G. N., Vagin, A. A. and Dodson, E. J. (1997) Refinement of macromolecular structures by the maximum-likelihood method. Acta Crystallogr D Biol Crystallogr. 53, 240-255

23 Emsley, P. and Cowtan, K. (2004) Coot: model-building tools for molecular graphics. Acta Crystallogr D Biol Crystallogr. 60, 2126-2132

24 Schuettelkopf, A. W. and van Aalten, D. M. (2004) PRODRG: a tool for highthroughput crystallography of protein-ligand complexes. Acta Crystallogr D Biol Crystallogr. 60, 1355-1363

25 Laskowski, R. A., MacArthur, M. W., Moss, D. S. and Thornton, J. M. (1993) PROCHECK: a program to check the stereochemical quality of protein structures. $J$ App Cryst. 283, 283-291

26 DeLano, W. L. (2002) The PyMOL Molecular Graphics System. DeLano Scientific, San Carlos, CA

27 Burmeister, W. P. (2000) Structural changes in a cryo-cooled protein crystal owing to radiation damage. Acta Crystallogr D Biol Crystallogr. 56, 328-341

28 Leiros, H. K., McSweeney, S. M. and Smalas, A. O. (2001) Atomic resolution structures of trypsin provide insight into structural radiation damage. Acta Crystallogr D Biol Crystallogr. 57, 488-497 
29 Padilla, J. E. and Yeates, T. O. (2003) A statistic for local intensity differences: robustness to anisotropy and pseudo-centering and utility for detecting twinning. Acta Crystallogr D Biol Crystallogr. 59, 1124-1130

30 Yeates, T. O. (1997) Detecting and overcoming crystal twinning. Methods Enzymol. 276, 344-358

31 Cruickshank, D. W. (1999) Remarks about protein structure precision, Acta Crystallogr D Biol Crystallogr. 55, 583-601 


\section{TABLES}

Table 1: Data collection and refinement statistics for the Eg5-STLC complex

\begin{tabular}{ll}
\hline & Eg5-STLC $^{1}$ \\
\hline Unit cell dimensions & $96.5,96.5,124.4$, \\
a, b, c, Y $\left(\AA,{ }^{\circ}\right)$ & 120 \\
Space group & $\mathrm{P}_{2}$ \\
Beamline / Detector & ID23-1 / Q315R \\
Molecules per asymmetric unit & 3 \\
Resolution range $(\AA)$ & $30-2.0$ \\
No. of unique reflections & 87,598 \\
Completeness $(\%)$ & $99.9(100.0)^{2}$ \\
Multiplicity & 5.8 \\
$R_{\text {sym }}(\%)$ & $8.9(40)$ \\
cl/ $\sigma(I)$ & $12.8(4.0)$ \\
Wilson $B\left(\AA^{2}\right) / D P I^{3}(\AA)$ & $27.2 / 0.14$ \\
\hline Refinement statistics & \\
\hline$R_{\text {work }} / \mathrm{R}_{\text {free }}(\%)$ & $15.9 / 21.8$ \\
Average B Factors & 35.9 \\
$\quad$ Overall & $33.6 / 37.7$ \\
$\quad$ Main Chain / Side Chain & \\
No. of. ADP / Inhibitor / Water & $3 / 3 / 1192$ \\
${ }^{3}$ r.m.s.d. in bond length $(\AA)$ & 0.0127 \\
r.m.s.d. in bond angle $\left({ }^{\circ}\right)$ & 1.61 \\
\hline
\end{tabular}

${ }^{1}$ The $S$ enantiomer was used for crystallization of the complex

${ }^{2}$ Values in parentheses pertain to the highest resolution shell

${ }^{3}$ DPI: Diffraction-component precision index [31]

${ }^{4}$ r.m.s.d. is the root-mean-square deviation from ideal geometry 


\section{FIGURE LEGENDS}

Figure 1: Overall ternary structure of Eg5 motor domain in complex with $\mathrm{Mg}^{2+} \mathrm{ADP}$ and STLC. Molecule A was used to generate these figures. (A) Stereoplot of the ternary ADP-Eg5-STLC complex with $\mathrm{Mg}^{2+} \mathrm{ADP}$ (red) and STLC (green) shown as ball-and-stick models. (B) Electron density (contoured at $\sigma=1.00$, coloured in blue) for STLC bound in the inhibitor-binding pocket.

Figure 2: Stereoplot showing STLC in the inhibitor-binding pocket and important hydrophobic, aromatic and hydrogen bond (represented by broken lines) interactions (within $4 \AA$ ) between STLC and the Eg5 main chain and side chain molecules, and structural water molecules represented by red spheres. Molecule A was used to generate this figure.

Figure 3: Stereoplots showing the overlay of the intermediate (molecule C, pink) and final inhibitor-bound states (molecule B, blue) of the ternary ADP-Eg5-STLC complex against native Eg5 (PDB ID: 1Il6, yellow). (A) Typical view of the inhibitor-binding pocket, showing the positions of Arg119, Trp127, Tyr211 and loop L5 before and after inhibitor-binding. The intermediate state (molecule $C$ ) is not shown because it has a similar conformation as the final inhibitor-bound state (molecule B). (B) Conformational changes in the helix $\alpha 3$ and Loop $L 9$ of the switch I region upon inhibitor binding. The intermediate state (molecule $C$ ) is again not shown because it has a similar conformation as the final inhibitor-bound state (molecule B). (C) The switch II cluster (helix a4, Loop L12 and helix a5) and the neck-linker region, enlarged and shown as insets, undergo conformational changes from native to intermediate (molecule C) to final inhibitor-bound state (molecule B). Helix a4 rotates by about $15^{\circ}$ to open up space for the ordered-to-ordered transition of the necklinker, which adopts a docked conformation in the final inhibitor-bound state. 
B Biochemical Journal Immediate Publication. Published on 30 Sep 2009 as manuscript BJ20091207

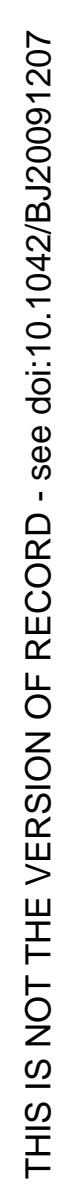

A
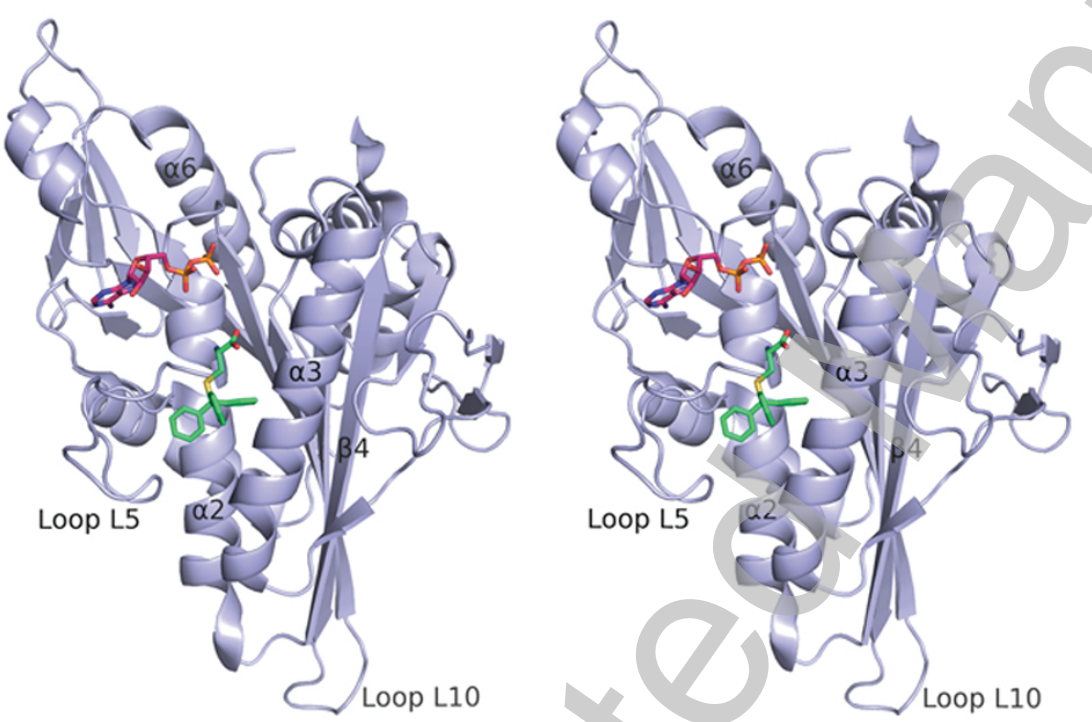

B

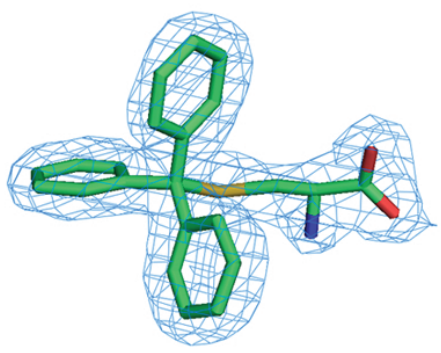

Licenced copy. Copying is not permitted, except with prior permission and as allowed by law.

(C) 2009 The Authors Journal compilation (c) 2009 Portland Press Limited 
B Biochemical Journal Immediate Publication. Published on 30 Sep 2009 as manuscript BJ20091207
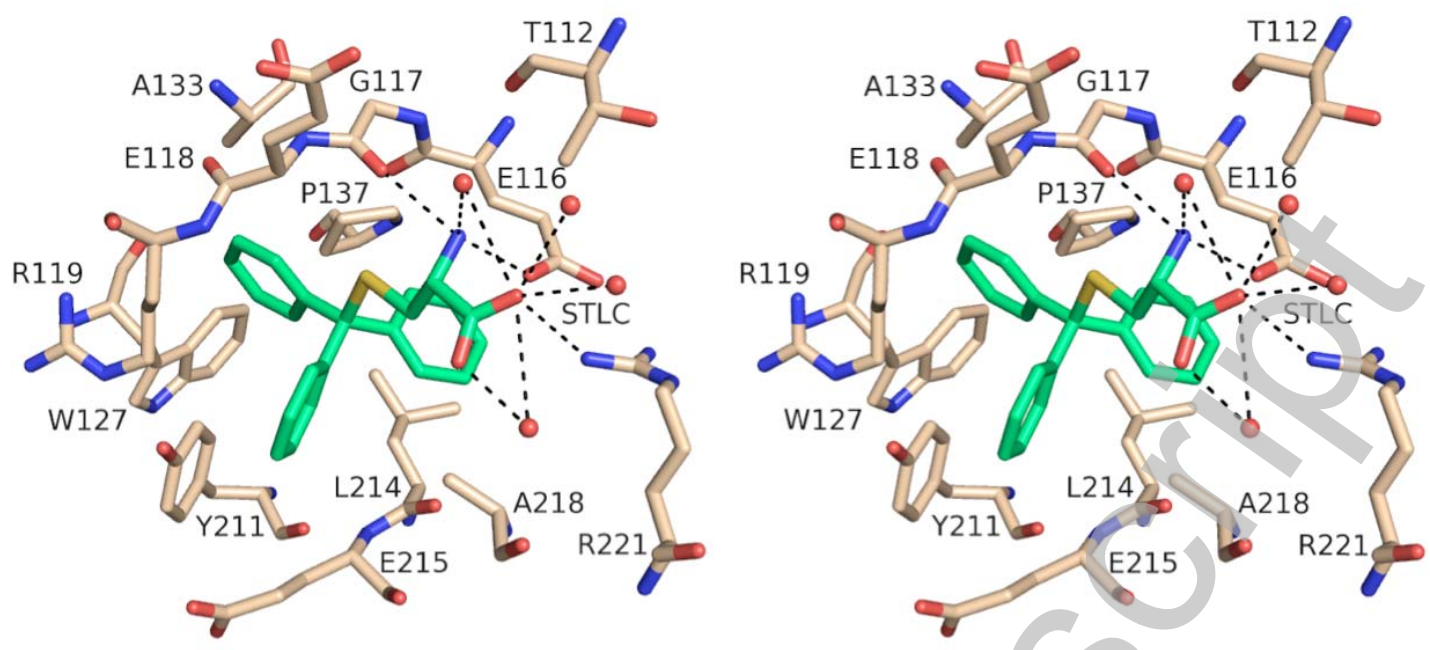

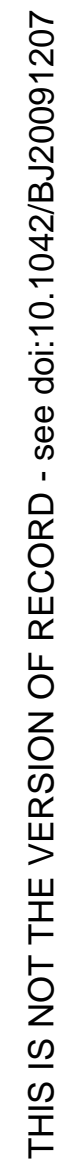

Licenced copy. Copying is not permitted, except with prior permission and as allowed by law. (C) 2009 The Authors Journal compilation (c) 2009 Portland Press Limited 

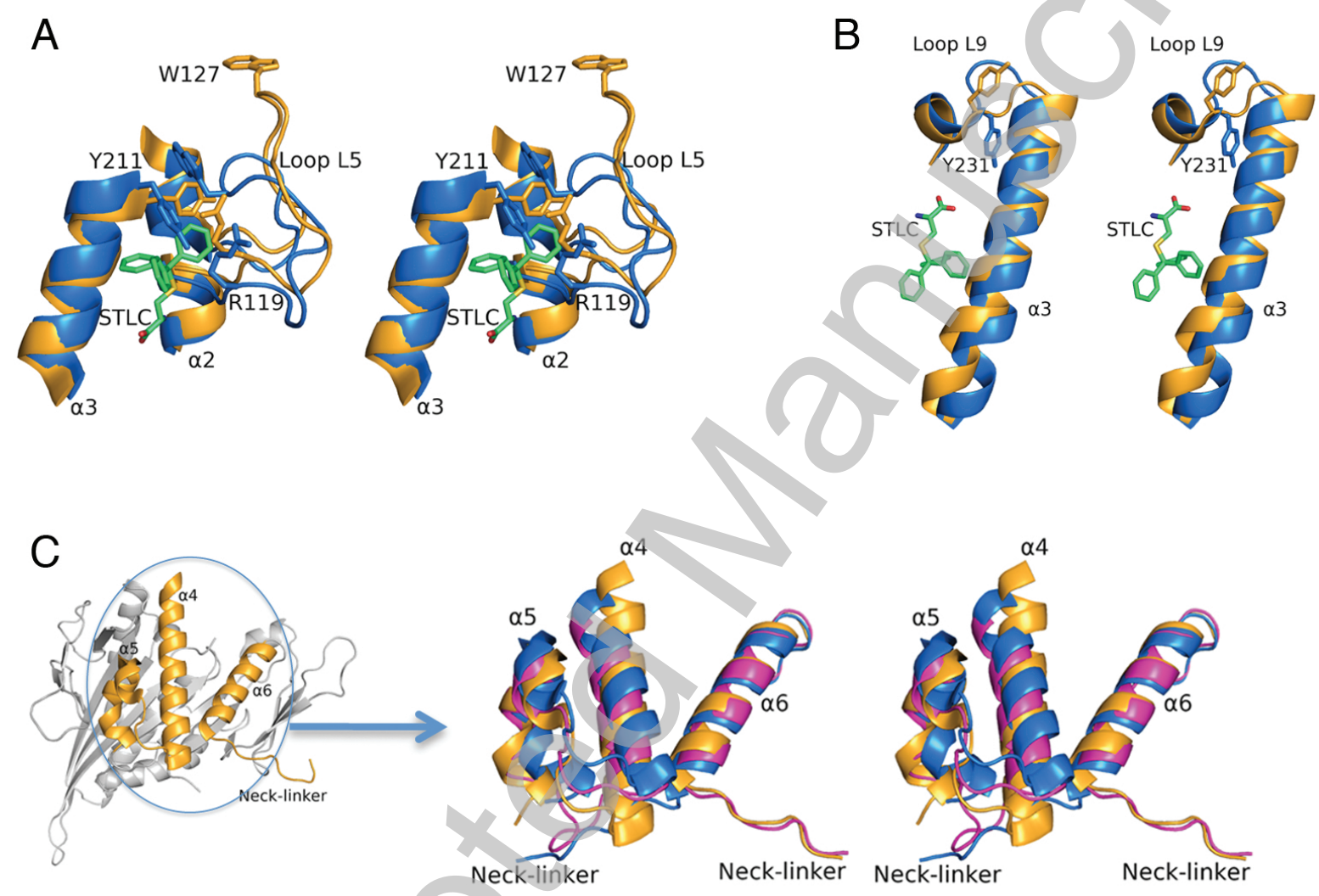

\section{Native Intermediate (Molecule C)}

Final inhibitor-bound state (Molecule $B$ ) 